ENCYCLOPEDDIE Encyclopédie berbère

BERBERE

$31 \mid 2010$

31 | Matmora - Meẓrag

\title{
Mauvais œil (Antiquité)
}

\section{J.-P. Laporte}

\section{OpenEdition}

Journals

Édition électronique

URL : http://journals.openedition.org/encyclopedieberbere/534

DOI : 10.4000/encyclopedieberbere.534

ISSN : 2262-7197

\section{Éditeur}

Peeters Publishers

\section{Édition imprimée}

Date de publication : 30 décembre 2010

Pagination : 4778-4783

ISBN : 978-90-429-2368-3

ISSN : 1015-7344

\section{Référence électronique}

J.-P. Laporte, « Mauvais œil (Antiquité) », Encyclopédie berbère [En ligne], 31 | 2010, document M74a, mis en ligne le 08 octobre 2020, consulté le 12 octobre 2020. URL : http://journals.openedition.org/ encyclopedieberbere/534; DOI : https://doi.org/10.4000/encyclopedieberbere.534

Ce document a été généré automatiquement le 12 octobre 2020

(C) Tous droits réservés 


\title{
Mauvais œil (Antiquité)
}

\author{
J.-P. Laporte
}

1 La crainte du «mauvais œil », plus superstitieuse que religieuse, occupait une grande place dans la vie antique. Elle semble se confondre avec la peur de l'« Invidus », ce que le mot français «Envieux» ne traduit que partiellement. Il s'agissait en fait d'un « regard malveillant et hostile », qui voulait du mal, qui voulait empêcher la réalisation de certains projets. L'Envieux était souvent qualifié de lividus, non pas très pâle, mais de la couleur jaune-bleu-noir d'un hématome, comme s'il avait reçu un coup.

2 La crainte de l'Envieux, très ancienne dans le monde méditerranéen (grec, romain, mais sans doute aussi libyque, tant ce souci est universel) fut souvent illustrée par de célèbres vers de Martial (Epigrammes, I, 40): Qui ducis uultus et non legis ista libenter omnibus inuideas, liuide, nemo tibi. «Toi qui fronces le sourcil et lis ces mots à contrecœur, puisses-tu, vilain jaloux, envier tout le monde sans que personne te porte envie ». Cette maxime connut un grand succès et fut souvent inscrite sur les ouvertures qu'on voulait dissuader l'envieux de franchir : piédroits et linteaux de portes (ou de fenêtres), mosaïques de seuil. Les exemples en Afrique sont nombreux. Sur une mosaïque de Lambiridi (Ballu, BCTH, 1919, p. 159 = Ann. Epig., 1921, 15), on lit : Invide vive et vide utpossis plura videre, «Envieux, vis et regarde, pour que tu puisses en voir plus encore » (sous entendu : « de ce que je construirai encore malgré toi »).

D'autres phrases ont été employées, notamment pour se féliciter de la réussite d'une entreprise malgré le 'mauvais œil'. Une dédicace sur mosaïque à Sullecthum, Salakta en Tunisie (Beschaouch 1968), célèbre, à grand renfort de réminiscences virgiliennes, l'achèvement (En perfecta...) de thermes et précise : Nisibus hic nostris prostratus libor (= livor) anhelat, "Par nos efforts ici terrassée, l'Envie est hors d'haleine ». De même à Thala, en Tunisie (CIL, VIII, 11683) : Hoc vide et vide utp[os]sisplura videre, "Regarde et regarde encore ceci (cet ouvrage terminé) pour voir encore d'autres choses (sous entendu : que tu n'auras pas réussi à empêcher) ». L'achèvement de l'œuvre est clairement interprété comme une défaite de l'Envieux, qui voulait, ici comme ailleurs, l'entraver. Le soulagement du responsable est souvent marqué par une formule du type et hoc factum est, « et ainsi, l'ouvrage est terminé », ainsi sur une mosaïque d'Uzitta. 
On menaçait aussi l'Invidus redouté. La phrase de Martial s'accompagnait parfois, en milieu païen, de menaces scatologiques (inuide cacas), ou obscènes (curiosos pedico), destinées à l'attaquer dans sa virilité. En l'absence de phrases, certains motifs précis faisaient l'affaire, notamment le phallus en érection (et quelquefois en éjaculation), qu'on retrouve d'ailleurs très souvent lui aussi sur des linteaux, des piédroits de pierre ou des mosaïques de seuil. Sur une stèle d'Auzia (moderne Sour el-Ghozlane, ex Aumale) élevée en mémoire d'un sous-officier de l'armée romaine, un beneficiarius (CIL, VIII, 9057= 20738), le «mauvais œil » est représenté tout simplement comme un œil assez naturaliste, mais ailé (fig. 1). Il est menacé de toutes parts. Un coq s'apprête à becqueter la paupière supérieure, un serpent placé à gauche parait mordre celle-ci d'un autre côté. En bas, la partie inférieure est attaquée par un scorpion qui y enfonce son dard, un escargot qui y applique ses cornes, enfin un lézard qui le mordille. Sur d'autres mosaïques, dont celle de Moknine (fig. 2) est la plus connue (Gauckler, Musée de Sousse, p. 36, pl. II, fig. 5 ; Inventaire des mosaïques de l'Afrique, $\mathrm{n}^{\circ} 78 \mathrm{~b}$ ), on voit le mauvais œil menacé par deux serpents et un phallus.

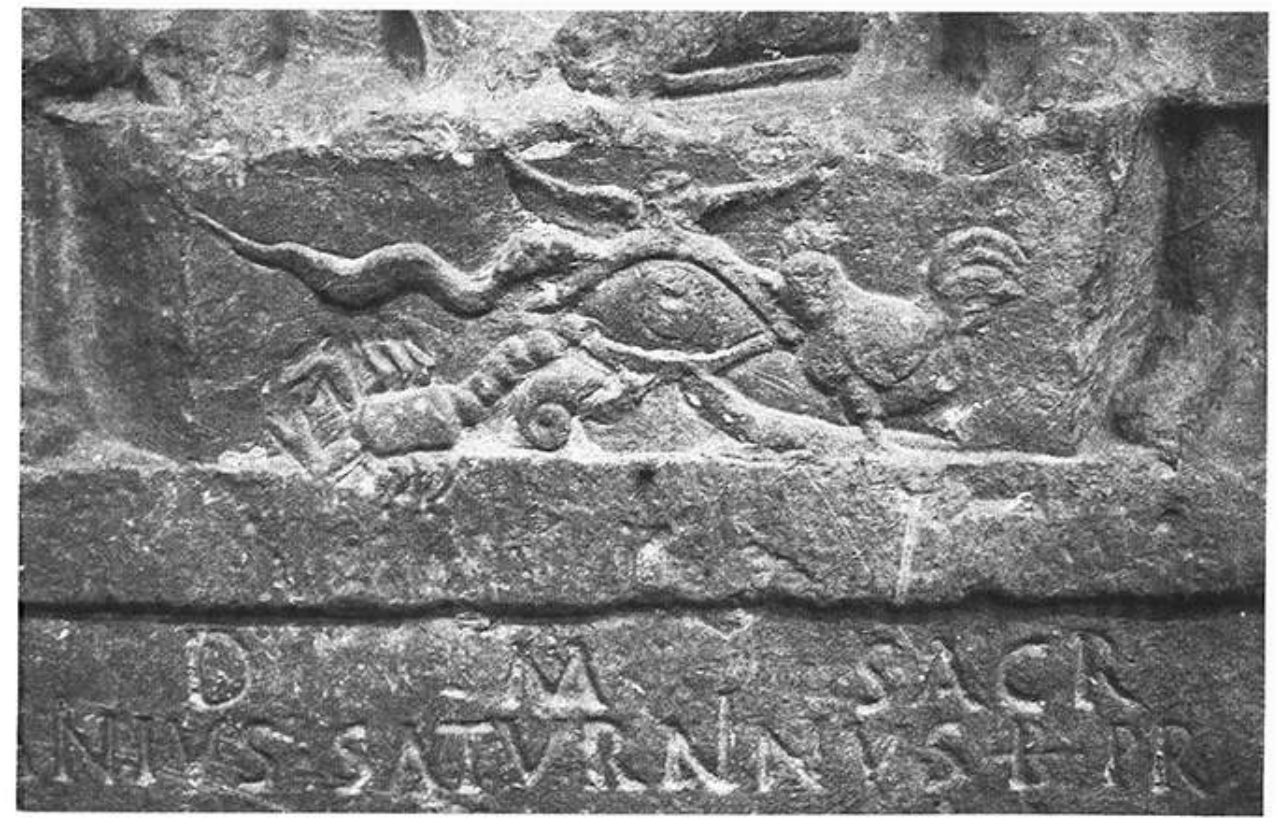

Fig. 1 : PARTIE de LA Stèle dU beneficiarius d'AUZIA. CIL VIII, CIL, VIII, 9057= 20738. ALger, MUSÉE DES ANTIQUités.

Cliché J.-P. Laporte, 1970. 


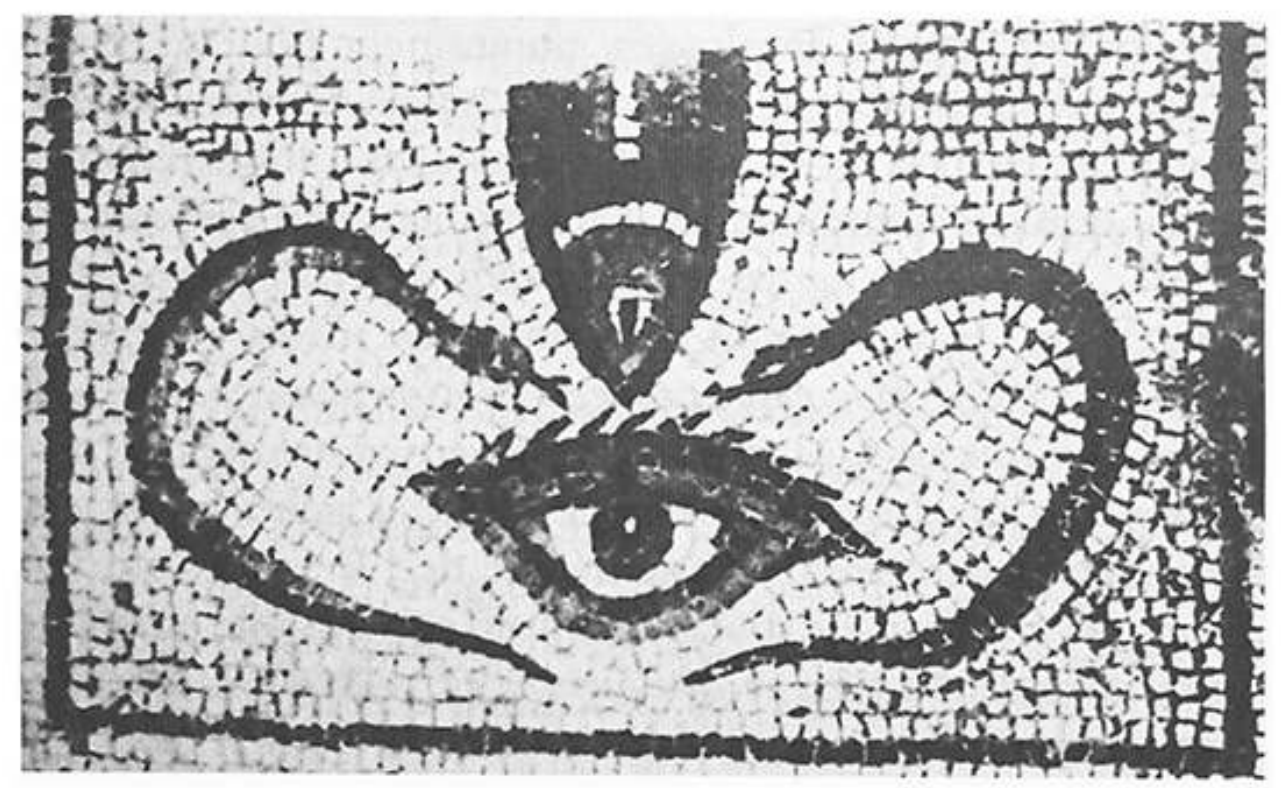

Fig. 2 : mosaïque de moKNine (MUSÉE de SOUSSE).

5 Les représentations du mauvais œil et des emblèmes qui le menaçaient étaient parfois fort stylisées, et nous ne savons pas toujours les reconnaître immédiatement dans des tableaux complexes. C'est ainsi qu'en haut d'une mosaïque d'El Haouria (fig. 3), représentant la dispute entre Athéna (symbolisant la sodalité des Palladii) et Poseïdon (celle des Pelagii) pour la suzeraineté de l'Attique (Beschaouch 1968), on lit l'inscription suivante : Invide livide, titula tanta, qu(a)e(m) adveserabas fieri, non posse perfecte (=perfecta) sunt, « Hé l'envieux livide! ces dédicaces d'édifices si considérables, qui, selon tes assertions, ne pouvaient voir le jour, les voilà menées à leur achèvement ». Dans la même mosaïque, deux exèdres portant des fuseaux dirigés vers un demi-cercle central pourraient passer pour une simple décoration géométrique, mais compte tenu de la formule qui précède, on reconnaît sans difficulté les défenses habituelles contre l'envieux : des phallus stylisés menaçant le mauvais œil réduit ici à un demi cercle.

Le milieu des jeux était particulièrement consommateur de maléfices. C'est ainsi que certains inscrivaient des malédictions envers tel ou tel cocher qu'ils voulaient voir perdre, notamment sur des tablettes de plomb (tabellae defixionum) qu'ils ensevelissaient subrepticement, parfois dans des tombes. Dans une forme adoucie, des mosaïques portaient des souhaits pour nous un peu elliptiques, mais à l'époque parfaitement clairs, ainsi à Thydrus / El Jem (Année épig., 1955, 84) : silentiu ut dormiant tauri, «silence, pour que les taureaux dorment» en fait "que la sodalité des Taurisci s'endorme » (sous entendu : pendant les jeux »). Une mosaïque de Thysdrus (Année. épig., $1995,1643)$ porte une scène complexe avec une chouette devant laquelle tombent des oiseaux, avec la formule Invidia rumpuntur aves neque noctua curat, « Les oiseaux crèvent d'envie, mais la chouette n'en a cure ». La chouette, symbole de Minerve, représentait ici une sodalité de dédicants de la ville de Thysdrus, dont la déesse était la protectrice, tandis que les oiseaux évoquaient une sodalité adverse dont nous ne connaissons pas le nom. 


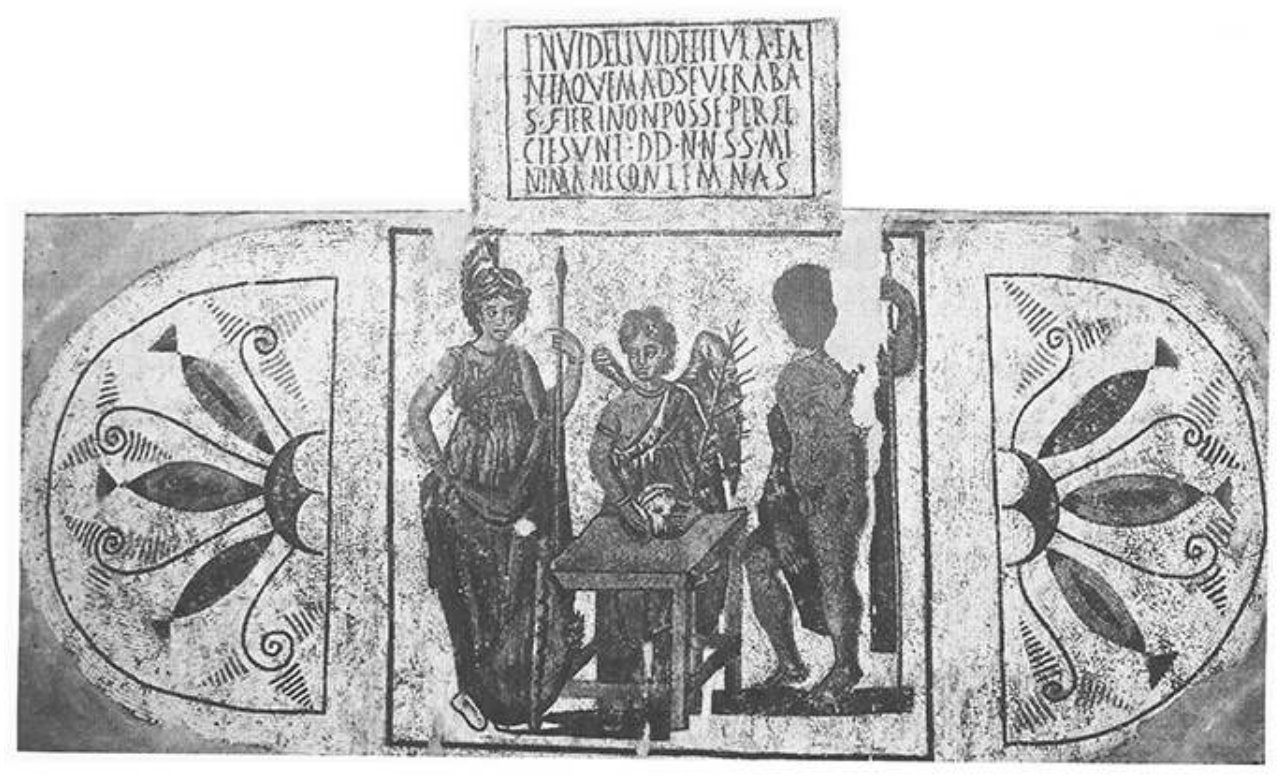

Fig. 3 : MOSAïque d'el haOURIA (MUSÉE de SOUSSE). d'APRÈs dUNBABIN 1978, PL. LXV.

7 La crainte de l'Envieux, du mauvais œil, était tellement ancrée dans l'imaginaire populaire, qu'elle ne s'affaiblit pas lors de l'arrivée du christianisme. Elle se concilia avec la foi chrétienne en adoptant des formes à peine adoucies. Le texte de Martial se retrouva certes en général privé des représentations anatomiques et des mentions obscènes de la période précédente. L'envie était en général considérée comme un péché bénin («véniel»), et saint Cyprien (évêque de Carthage de 248 à 258) commençait comme suit son De zelo et livore: Zelare quod bonum videas et invidere melioribus leve apud quosdam et modicum crimen videtur, "Jalouser le bien que l'on voit et envier ceux qui valent mieux que soi [ou : 'ce qui va mieux ailleurs'] passe auprès de certains pour une faute légère et sans gravité ». Pour lui, ce péché était grave. L'Inuidus fut, sinon identifié avec le diable, du moins nettement mis en parallèle. Toujours pour saint Cyprien (De zelo et livore, IV), c'est par l'Envie que le démon s'était perdu lui-même à l'origine du monde, et il n'avait trouvé sur terre que trop d'imitateurs : l'Envie était la source d'une foule de calamités, une pépinière d'incalculables désordres. À I'djâz (Syrie), une prière proclamait: «Je ne redoute pas les machinations du malin démon, ni l'œil mauvais et haineux de l'homme»"

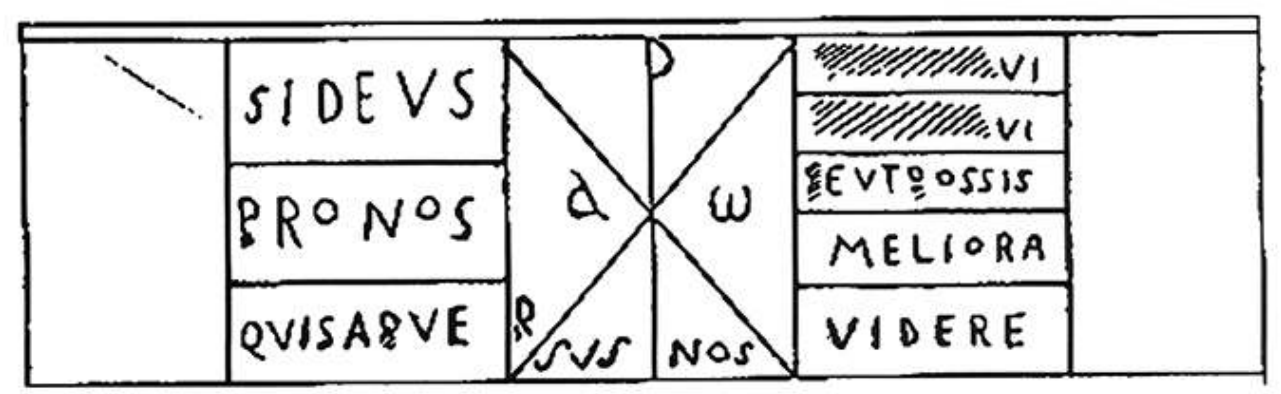

Fig. 4 : LINTEAU ChRÉtIEN DE TIgZIRT. D'APRÈs UN RELEVÉ D'ALAIN HUS, 1951, APUD LAPORTE, 1994.]

8 On retrouve par ailleurs sur de nombreuses inscriptions chrétiennes d'Afrique une phrase de saint Paul (Ad. Rom., VIII, 31): Si Deus / pro nobis /, quis aduersus nos ?, « si Dieu est avec nous, qui (sera) contre nous". Saint Paul donnait bien sûr à cette phrase une dimension spirituelle. Nombre de chrétiens y ont vu tout autre chose, et ont exprimé 
par là des sentiments de rejet et d'exclusion. Paul Monceaux l'a interprétée comme un indice de donatisme, position sans aucun doute excessive. Paul-Albert Février a estimé qu'il s'agissait d'un quelconque texte de lutte. Nous pouvons aller plus loin grâce à un linteau chrétien épigraphe de Tigzirt (fig. 4) découvert par Alain Hus en 1951 (Laporte 1994) qui porte deux inscriptions de part et d'autre d'un chrisme. À gauche la phrase de saint Paul: Si Deus / pro no(bi)s /, quis aduersus nos? À droite, la phrase classique à l'époque païenne : [inuide], ui/[ue et] ui/[d]e ut possis / meliora / uidere. L'association de la formule toute païenne de Martial et de la phrase de saint Paul, en apparence bien chrétienne, montre que cette dernière était devenue une forme christianisée du même souci, la crainte de l'Envieux, auquel il convenait d'interdire l'entrée. Reste le chrisme, qui, bien que pouvant être interprété isolément, n'en est pas moins étroitement encadré sur le linteau de Tigzirt par les deux formules classiques, païenne et chrétienne, contre l'Invidus. Il est clair qu'il a ici, lui-aussi, une valeur " apotropaïque » (= protectrice). Le cas n'est pas isolé. Comme l'indique clairement une inscription datée de 546 sur une maison de Sabbâ (Syrie) citée par Dom Leclercq : «la croix s'y trouvant placée en tête, le mauvais cil ne prévaudra pas ».

\section{BIBLIOGRAPHIE}

BESCHAOUCH A., «Echec à l'envieux, d'après une inscription métrique sur mosaïque découverte dans des thermes à Sullecthum en Tunisie ", Atti della Accademia Nazionale dei Lincei, Rendiconti, série VIII, Scienze morali, storiche filologiche, t. XXIII, 1968, p. 59-68 et pl. I-II.

BESCHAOUCH A., «Référents grecs, expression latine : à propos de la culture des sodalités africaines », dans La Méditerranée d'une rive à l'autre, Paris, De Boccard, 2007, p. 185-199, notamment p. $196-198$.

CYPRIEN (saint), De zelo et livore, IV, éd. M. Poirier, Sources chrétiennes, $\mathrm{n}^{\circ} 519$.

DUNBABIN K. M. D., The mosaics of Roman North Africa, Oxford, 1978, p. 261 (El Haouria) et pl. 165 (cf. également p. 161-163).

FÉVRIER P.-A., « Les sources épigraphiques et archéologiques de l'histoire religieuse des provinces orientales de l'Afrique romaine ", Corso Ravenna, 1972, p. 135, n. 20.

LAPORTE J.-P., « Tigzirt : saint Paul contre l'Inuidus », L'Afrique, la Gaule, la Religion à l'époque romaine. Mélanges Le Glay = Collection Latomus, 226, 1994, p. 285-287, pl. XLV.

LECLERCQ Dom. H., s.v. magie, DACL, X, 1931, col. 1112, pour la lutte contre l'envoûtement.

MARCILLET-JAUBERT J., « Un propriétaire ombrageuX », Epigraphica, XXXIII, 1975, p. 153-158.

MARROU H.-I., Deux inscriptions chrétiennes, BAA, III, 1968, p. 348-350, II, Le linteau d'Henchir Zoura.

MONCEAUX P., «L'épigraphie donatiste », Revue de Philologie, XXXIII, 1909, p. 112-161, repris dans Histoire Littéraire de l'Afrique chrétienne, IV, 1912, p. 437-484. 
INDEX

Mots-clés : Antiquité, Croyances, Ethnologie, Ethnographie, Histoire, Maurétanie, Peuplement, Population 